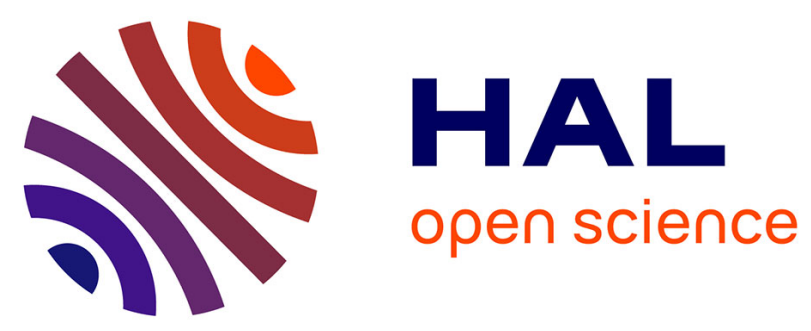

\title{
Effective spin- 12 description of transverse-field-induced random fields in dipolar spin glasses with strong single-ion anisotropy
}

S. M A Tabei, F. Vernay, M. Gingras

\section{- To cite this version:}

S. M A Tabei, F. Vernay, M. Gingras. Effective spin- 12 description of transverse-field-induced random fields in dipolar spin glasses with strong single-ion anisotropy. Physical Review B: Condensed Matter and Materials Physics (1998-2015), 2008, 77 (1), 10.1103/PhysRevB.77.014432 . hal-02145564

\author{
HAL Id: hal-02145564 \\ https://hal.science/hal-02145564
}

Submitted on 3 Jun 2019

HAL is a multi-disciplinary open access archive for the deposit and dissemination of scientific research documents, whether they are published or not. The documents may come from teaching and research institutions in France or abroad, or from public or private research centers.
L'archive ouverte pluridisciplinaire HAL, est destinée au dépôt et à la diffusion de documents scientifiques de niveau recherche, publiés ou non, émanant des établissements d'enseignement et de recherche français ou étrangers, des laboratoires publics ou privés. 


\title{
Effective spin- $\frac{1}{2}$ description of transverse-field-induced random fields in dipolar spin glasses with strong single-ion anisotropy
}

\author{
S. M. A. Tabei, ${ }^{1}$ F. Vernay, ${ }^{1}$ and M. J. P. Gingras ${ }^{1,2,3}$ \\ ${ }^{1}$ Department of Physics and Astronomy, University of Waterloo, Waterloo, Ontario, Canada N2L $3 G 1$ \\ ${ }^{2}$ Department of Physics and Astronomy, University of Canterbury, Private Bag 4800, Christchurch, New Zealand \\ ${ }^{3}$ Canadian Institute for Advanced Research, 180 Dundas Street W., Toronto, Ontario, Canada M5G 1Z8
}

(Received 16 August 2007; published 22 January 2008)

\begin{abstract}
We present analytical and numerical evidence for the validity of an effective $S_{\text {eff }}=\frac{1}{2}$ approach to the description of random field generation in $S \geqslant 1$, and especially for $S=1$, dipolar spin-glass models with strong uniaxial Ising anisotropy and subject to weak external magnetic field $B_{x}$ transverse to the Ising direction. Explicitly $B_{x}$-dependent random fields are shown to naturally emerge in the effective low-energy description of a microscopic $S=1$ toy model. We discuss our results in relation to recent theoretical studies pertaining to the topic of $B_{x}$-induced random fields in $\mathrm{LiHo}_{x} \mathrm{Y}_{1-x} \mathrm{~F}_{4}$ magnetic materials with the $\mathrm{Ho}^{3+}$ Ising moments subject to a transverse field. We show that the $S_{\text {eff }}=\frac{1}{2}$ approach is able to capture both the qualitative and quantitative aspects of the physics at small $B_{x}$, giving results that agree with those obtained using conventional secondorder perturbation theory.
\end{abstract}

DOI: 10.1103/PhysRevB.77.014432

PACS number(s): 75.10.Jm, 05.50.+q, 75.10.Nr, 75.50.Lk

\section{INTRODUCTION}

In condensed matter physics systems with strongly interacting quantum mechanical degrees of freedom, it is often a challenge to explain physical phenomena from a truly firstprinciples atomistic point of view. In systems where there are high energy scales well separated from a low-energy sector, effective low-energy theories offer the advantage of a reformulation of the problem with an exponentially smaller Hilbert space. A well-known and topical example where such an approach is used is in the derivation of an effective spin-only model starting from a Hubbard model describing electrons hopping on a lattice. It is commonly accepted that the lowenergy magnetic excitations of a Hubbard model with a large Coulomb repulsion $U$ are easier to investigate within an effective spin Hamiltonian. ${ }^{1-3}$ Generally speaking, the only requirement to be able to derive an effective model is to have a small parameter, which is $t / U$ in the previous example, where $t$ is the nearest-neighbor hopping constant.

In many magnetic materials, the ground-state degeneracy of the otherwise free magnetic ions can be partially lifted by electrostatic and covalent interactions due to the surrounding atoms - the so-called crystal field effect. In a number of situations, the energy scales associated with the spin-spin interactions are much smaller than the energy gap between the single-ion ground state and the excited crystal field states. In such cases, one can often, as a first approximation, neglect the high-energy states and reduce the relevant Hilbert space to a much smaller subspace of low-energy states. In this paper, we discuss the quantitative validity of an effective low-energy theory description of a model of anisotropic (dipolar) spin glass in strong single-ion anisotropy in the presence of a transverse field. The study of this model is inspired by the phenomena displayed by the disordered $\mathrm{LiHo}_{x} \mathrm{Y}_{1-x} \mathrm{~F}_{4}$ Ising magnetic material when subject to an external magnetic field $B_{x}$ applied perpendicular to the Ising direction of the $\mathrm{Ho}^{3+}$ magnetic moments.

The $\mathrm{LiHo}_{x} \mathrm{Y}_{1-x} \mathrm{~F}_{4}$ magnetic material exhibits many interesting magnetic behaviors. ${ }^{4-10}$ The magnetic properties of
$\mathrm{LiHo}_{x} \mathrm{Y}_{1-x} \mathrm{~F}_{4}$ are due to the $\mathrm{Ho}^{3+}$ ions. The single-ion ground state of $\mathrm{Ho}^{3+}$ is a doublet, while the first excited state is at an energy of $\sim 11 \mathrm{~K}$ above the ground state. ${ }^{11,12}$ The most relevant interactions between the magnetic $\mathrm{Ho}^{3+}$ ions are magnetic dipole-dipole interactions. ${ }^{13}$ Since the maximum strength of the dipolar interactions is for nearest-neighbor separation and is approximately $0.31 \mathrm{~K}$, collective behavior in this material occurs at temperatures less than $O(1 \mathrm{~K})$, where only the ground doublet is significantly thermally populated. Consequently, the cooperative phenomena and the low-temperature properties of this material in zero applied magnetic field should be well captured by a model with effectively spin- $\frac{1}{2}$ degrees of freedom. ${ }^{13,14}$ For example, in zero applied magnetic field, the system can be recast as a diluted dipolar Ising model with the low-temperature phase being either a ferromagnet or a spin glass depending on the concentration $x$ of magnetic ions. ${ }^{4,15-17}$ On the other hand, for $x=1$ and with a magnetic field $B_{x}$ applied perpendicular to the crystallographic Ising $c$-axis direction, $\mathrm{LiHoF}_{4}$ has been advocated as one of the rare physical realizations ${ }^{14}$ of the transverse field Ising model (TFIM). ${ }^{18-22}$ Yet it is only relatively recently that a somewhat rigorous justification of a TFIM description of $\mathrm{LiHoF}_{4}$ in nonzero $B_{x}$ has been put forward. ${ }^{13}$ However, over the past 20 years, and until very recently, several experimental studies had found the behavior of $\mathrm{LiHo}_{x} \mathrm{Y}_{1-x} \mathrm{~F}_{4}\left(x<1, B_{x} \neq 0\right)$ paradoxical, as we now discuss.

One may have naively expected that the application of a transverse magnetic field in $\mathrm{LiHo}_{x} \mathrm{Y}_{1-x} \mathrm{~F}_{4}$ would allow one to straightforwardly explore the physics of the TFIM in either a diluted ferromagnet or a spin glass, depending on the concentration $x$. However, the situation for $x<1$ is quite a bit more complicated. ${ }^{5}$ For example, when $B_{x}=0$, $\mathrm{LiHo}_{0.167} \mathrm{Y}_{0.833} \mathrm{~F}_{4}$ displays a conventional spin-glass phase transition $^{5,16,17}$ with a nonlinear magnetic susceptibility $\chi_{3}$ diverging at the spin-glass transition temperature $T_{g}$ as $\chi_{3}(T) \propto\left(T-T_{g}\right)^{-\gamma}$, as in ordinary spin-glass materials. ${ }^{23}$ However, as $B_{x}$ is increased from zero, $\chi_{3}(T)$ becomes steadily 
less singular and there appears to be an absence of a $B_{x}$-induced quantum critical phase transition between a paramagnet and a spin-glass state. ${ }^{5}$ This puzzling experimental behavior had been tentatively interpreted as due to a firstorder transition near the $T=0$ quantum phase transition. 5,24 However, very recent and independent theoretical investigations ${ }^{25-27}$ have instead proposed that the microscopic origin of the "quenching" of the paramagnetic to spin glass transition as $B_{x}$ is turned on is due to the generation of random fields that destroy the spin-glass phase.

The authors of Ref. 26 used an effective $S_{\text {eff }}=\frac{1}{2}$ theory, very similar to the one developed for pure $\mathrm{LiHoF}_{4},{ }^{13}$ to expose how random fields develop in a microscopic model of $\mathrm{LiHo}_{x} \mathrm{Y}_{1-x} \mathrm{~F}_{4}$ in nonzero $B_{x}$. In particular, Ref. 26 showed how the nonlinear susceptibility $\chi_{3}$ becomes progressively less singular as $B_{x}$ is increased. Also motivated by the phenomena displayed by $\mathrm{LiHo}_{x} \mathrm{Y}_{1-x} \mathrm{~F}_{4}$, Schechter and collaborators $^{25,27,28}$ recently investigated in a series of papers the general phenomenology of induced random fields in $\mathrm{LiHo}_{x} \mathrm{Y}_{1-x} \mathrm{~F}_{4}$. To do so, they considered in Refs. 25 and 27 an easy-axis spin- $S(S \geqslant 1)$ dipolar spin-glass toy model Hamiltonian $\mathcal{H}$ in presence of a nonzero $B_{x}$. By using second-order perturbation theory, invoking the scaling droplet picture of Fisher and Huse for spin glasses, ${ }^{29}$ and using an Imry-Ma type argument, ${ }^{30}$ Schechter et $a l .{ }^{25,27}$ calculated the finite energy $\delta E$ required to flip the spins within a spin-glass droplet. In their calculations, they found a limit on how large the spin-glass correlation length $\xi$ can grow as the system is cooled from the paramagnetic phase. The behavior of the system, and the corresponding $\delta E$, is found to be analogous to that of a spin glass in a random magnetic field, which, according to the droplet model, does not show a spin-glass transition in nonzero field. ${ }^{31}$ As a result, Refs. 25 and 27 argue that no spin-glass transition can occur in a dipolar spin glass where random off-diagonal dipolar interactions and an applied transverse magnetic field are simultaneously at play.

The results of both Refs. 25 and 27 and Ref. 26 derive from the notion that the applied transverse field generates effective random fields. The average magnetization along the direction of the applied transverse field induces the random fields via the off-diagonal part of the dipolar interactions, which couple the Ising $\hat{z}$ component with the perpendicular $\hat{x}$ and $\hat{y}$ components. However, what is not clear is how the random fields in these two calculations are related or if, in fact, they are equivalent. In their studies, the authors of Refs. 25 and 27 argued, correctly, that consideration of a model with large spin $(S \geqslant 1)$ is crucial in order to understand the weak-field response of the spin-glass phase in either their toy model $\mathcal{H}$ or $\mathrm{LiHo}_{x} \mathrm{Y}_{1-x} \mathrm{~F}_{4}$. Furthermore, exact diagonalization results of an $S=1$ dipolar spin glass model with easyaxis anisotropy provided further quantitative support to the theoretical arguments as to the scaling behavior of $\delta E$ with both $B_{x}$ and the number of spins in the system. ${ }^{25,27}$ Reference 27 also considered an effective anisotropic spin- $\frac{1}{2}$ dipolar Ising model in a transverse field, but with the off-diagonal dipolar interactions rescaled compared to the longitudinal Ising coupling. ${ }^{26}$ For that model, their numerical results did not conform with those obtained for the "bare" (high-energy) anisotropic $S=1$ model. ${ }^{25,27}$ Partially on the basis of those results, and seemingly confirming a previous suggestion, ${ }^{25}$ Ref. 27, concludes that an effective spin- $\frac{1}{2}$ model, such as that used in Ref. 26, is not sufficient to capture the physics in the small- $B_{x}$ regime compared to the bare microscopic (large-spin) anisotropic dipolar spin-glass model $\mathcal{H}$.

Considering a perspective beyond the specific problem of $\mathrm{LiHo}_{x} \mathrm{Y}_{1-x} \mathrm{~F}_{4}$, one could interpret the conclusion of Refs. 25 and 27 regarding the inadequacies of an effective spin- $\frac{1}{2}$ model to describe $\mathrm{LiHo}_{x} \mathrm{Y}_{1-x} \mathrm{~F}_{4}$ in $B_{x} \neq 0$, as a counterexample of the precise quantitative usefulness of effective lowenergy theories for quantum $N$-body systems. It is therefore of interest to investigate with some scrutiny the mathematical justification for an effective spin- $\frac{1}{2}$ model for $\mathrm{LiHo}_{x} \mathrm{Y}_{1-x} \mathrm{~F}_{4}$ with $B_{x} \neq 0$. This is the purpose of the present paper. More specifically, the question that we ask here is to what extent the explicitly manifest random fields derived in an effective low-energy theory, as in Ref. 26, are related to the random-field-like effects at play in perturbation theories, as used in Refs. 25 and 27. Below we show, via a derivation of an effective low-energy $S_{\text {eff }}=\frac{1}{2}$ Hamiltonian for anisotropic dipolar glasses, that effective random longitudinal fields emerge naturally in the $S_{\text {eff }}=\frac{1}{2}$ model. On the basis of analytical calculations and exact diagonalizations, we highlight the fact that an $S_{\text {eff }}=\frac{1}{2}$ Hamiltonian properly derived from an $S=1$ high-energy toy model $\mathcal{H}$, such as the one proposed in Refs. 25 and 27 [see Eq. (1) in Sec. II], is a quantitatively valid and controlled approach to this problem.

The paper is organized as follows. We discuss in Sec. II an anisotropic spin- $S$ dipolar Hamiltonian as a simplified model displaying some of the key physics of the $\mathrm{LiHo}_{x} \mathrm{Y}_{1-x} \mathrm{~F}_{4}$ material in a transverse field. We then show in Sec. III how to derive from it an effective $S_{\text {eff }}=\frac{1}{2}$ Hamiltonian to lowest order. We present in Sec. IV results from exact diagonalization calculations that compare the $S=1$ and the $S_{\text {eff }}=\frac{1}{2}$ models, and which directly confirm the quantitative validity of the effective Hamiltonian approach. Section $\mathrm{V}$ concludes the paper.

\section{ANISOTROPIC SPIN HAMILTONIAN}

The $\mathrm{Ho}^{3+}$ ion is characterized by a very large hyperfine interaction between the electronic and nuclear moments, and the effects of this strong interaction plays an important role in a number of $\mathrm{Ho}^{3+}$-based magnetic materials. ${ }^{8,12,14,33,34}$ In particular, in $\mathrm{LiHoF}_{4}$, it leads to a significant increase of the zero-temperature critical transverse field for the dipolar ferromagnet to quantum paramagnet transition. ${ }^{13,14}$ It also plays an important role in setting the relevant critical transverse magnetic field scale in dilute $\mathrm{LiHo}_{x} \mathrm{Y}_{1-x} \mathrm{~F}_{4} \cdot{ }^{35}$ In this paper, however, we are specifically interested in the general phenomenology of random fields along the Ising spin directions generated by a small applied transverse field rather than obtaining a precise quantitative description of $\mathrm{LiHo}_{x} \mathrm{Y}_{1-x} \mathrm{~F}_{4}$. In this specific context, we therefore neglect the role of hyperfine interactions. Also neglecting the hyperfine interactions, Schechter et al. ${ }^{25,27}$ proposed a generic anisotropic spin- $S$ toy model Hamiltonian with long-range dipolar interactions 


$$
\mathcal{H}=-D \sum_{i}\left[\left(S_{i}^{z}\right)^{2}-S^{2}\right]-\sum_{i \neq j}\left(\frac{1}{2} V_{i j}^{z z} S_{i}^{z} S_{j}^{z}+V_{i j}^{z x} S_{i}^{z} S_{j}^{x}\right)-B_{x} \sum_{i} S_{i}^{x} .
$$

This Hamiltonian is a simplified model that preserves the basic characteristics of the proposed microscopic Hamiltonian ${ }^{13,26}$ for $\mathrm{LiHo}_{x} \mathrm{Y}_{1-x} \mathrm{~F}_{4}$. In the absence of an external field, individual $\mathrm{Ho}^{3+}$ spins have an Ising-like groundstate doublet with a large energy gap between the lowest excited state and the ground doublet. Also, for $S=1$, the excited state of the model in Eq. (1) is a singlet, as for $\mathrm{Ho}^{3+}$ in $\mathrm{LiHo}_{x} \mathrm{Y}_{1-x} \mathrm{~F}_{4}{ }^{11,12} \mathrm{Here}, i, j$ are the positions of the randomly positioned magnetic moments. $V_{i j}^{\mu \nu}$ denotes the random longrange dipolar interaction between the spins, where $V_{i j}^{z z}$ stands for the Ising interaction and $V_{i j}^{z x}$ stands for the off-diagonal interaction $\left(V_{i j}^{\mu \nu}=V_{i j}^{\nu \mu}\right.$ for dipolar interactions). $D>0$ is the anisotropy constant mimicking the crystal field. For $B_{x}=0$, the ground state (GS) of a single spin is doubly degenerate with $S^{z}= \pm S$. The corresponding states of the doublet are denoted $|S\rangle$ and $|-S\rangle$. The first excited states have $S^{z}$ $= \pm(S-1)$ and energy $\Omega_{0} \equiv(2 S-1) D$ with respect to the ground-state doublet and with the corresponding states denoted as $| \pm(S-1)\rangle$. Ignoring momentarily the $V_{i j}^{\mu \nu}$ interactions, the Zeeman term $-B_{x} \Sigma_{i} S_{i}^{x}$ lifts the GS degeneracy of the $| \pm S\rangle$ ground doublet, resulting in two new lowest-energy states $\left|\alpha\left(B_{x}\right)\right\rangle$ and $\left|\beta\left(B_{x}\right)\right\rangle$, with corresponding energies $E_{\alpha}\left(B_{x}\right)$ and $E_{\beta}\left(B_{x}\right)$, and with an energy gap

$$
\Delta\left(B_{x}\right)=E_{\alpha}\left(B_{x}\right)-E_{\beta}\left(B_{x}\right)
$$

between them. For $B_{x} \ll \Omega_{0}$, to leading order in perturbation theory, the gap $\Delta\left(B_{x}\right)$ is proportional to $\left(B_{x}\right)^{2 S} \cdot{ }^{36}$

Invoking the spin-glass droplet scaling picture of Fisher and Huse, ${ }^{29}$ and using an Imry-Ma ${ }^{30}$ type argument, one can calculate the energy required to flip a spin-glass droplet of size $L$ containing $N \sim L^{d}$ spins, with $d$ the number of space dimensions (here $d=3$ ). This energy cost is due to the perturbative quantum term $\mathcal{H}_{\perp} \equiv-\Sigma_{i \neq j} V_{i j}^{z x} S_{i}^{z} S_{j}^{x}-B_{x} \Sigma_{i} S_{i}^{x}$, which does not commute with the classical term $\mathcal{H}_{\|}=-D \Sigma_{i}\left[\left(S_{i}^{z}\right)^{2}\right.$ $\left.-S^{2}\right]-\frac{1}{2} \Sigma_{i \neq j} V_{i j}^{z z} S_{i}^{z} S_{j}^{z}$. Considering first only $\mathcal{H}_{\|}$, and invoking the droplet picture of only two distinct ground states, ${ }^{29}\left|\Phi_{S}\right\rangle$ and $\left|\widetilde{\Phi}_{S}\right\rangle$ denote the collective (doubly degenerate) Ising spin-glass ground states of the system. These two ground states are related by the global $S_{i}^{z} \rightarrow-S_{i}^{z}$ symmetry, where each spin is in either its $|+S\rangle$ state or its $|-S\rangle$ state. As discussed in Refs. 25 and 27, nonzero $\mathcal{H}_{\perp}$ lifts the ground-state degeneracy, as we now review in order to make contact with the results presented below in Secs. III and IV.

The lowest-energy excited states (above the otherwise two degenerate $\left|\Phi_{S}\right\rangle$ and $\left|\tilde{\Phi}_{S}\right\rangle$ ground states) are $\left|\phi_{(S-1)}^{k}\right\rangle$ and $\left|\widetilde{\phi}_{(S-1)}^{k}\right\rangle$ states, in which the $k$ th spin has its $S^{z}$ quantum value changed from $+S$ to $+(S-1)$ or from $-S$ to $-(S-1)$. Using standard second-order degenerate perturbation theory, ${ }^{37}$ and considering only excitations to the (intermediate excited) $\left|\phi_{(S-1)}\right\rangle$ and $\left|\widetilde{\phi}_{(S-1)}^{k}\right\rangle$ states, the fluctuation-induced energy difference between $\left|\Phi_{S}\right\rangle$ and $\left|\widetilde{\Phi}_{S}\right\rangle$ is

$$
\delta E=\sqrt{\left(H_{\Phi_{S}, \Phi_{S}}-H_{\tilde{\Phi}_{S}}, \tilde{\Phi}_{S}\right)^{2}+4 \mid H_{\Phi_{S}}, \tilde{\Phi}_{S}{ }^{2}},
$$

where

$$
\begin{aligned}
& H_{\Phi_{S}, \Phi_{S}}=-\frac{1}{\Omega_{0}} \sum_{k}\left|\left\langle\Phi_{S}\left|H_{\perp}\right| \phi_{(S-1)}^{k}\right\rangle\right|^{2}, \\
& H_{\tilde{\Phi}_{S}, \tilde{\Phi}_{S}}=-\frac{1}{\Omega_{0}} \sum_{k}\left|\left\langle\widetilde{\Phi}_{S}\left|H_{\perp}\right| \widetilde{\phi}_{(S-1)}^{k}\right\rangle\right|^{2},
\end{aligned}
$$

and

$$
\begin{aligned}
H_{\Phi_{S}, \tilde{\Phi}_{S}=} & -\frac{1}{\Omega_{0}} \sum_{k}\left\langle\Phi_{S}\left|H_{\perp}\right| \phi_{(S-1)}^{k}\right\rangle\left\langle\phi_{(S-1)}^{k}\left|H_{\perp}\right| \widetilde{\Phi}_{S}\right\rangle \\
& +\left\langle\Phi_{S}\left|H_{\perp}\right| \widetilde{\phi}_{(S-1)}^{k}\right\rangle\left\langle\widetilde{\phi}_{(S-1)}^{k}\left|H_{\perp}\right| \widetilde{\Phi}_{S}\right\rangle,
\end{aligned}
$$

where we have taken the ground-state energy to be zero. Since $\left\langle\Phi_{S}\left|H_{\perp}\right| \widetilde{\phi}_{(S-1)}^{k}\right\rangle=\left\langle\widetilde{\Phi}_{S}\left|H_{\perp}\right| \phi_{(S-1)}^{k}\right\rangle=0$, we have $H_{\Phi_{S}}, \tilde{\Phi}_{S}$ $=0$. Subtracting $H_{\tilde{\Phi}_{S}}, \tilde{\Phi}_{S}$ from $H_{\Phi_{S}, \Phi_{S}}$, only the odd terms in $B_{x}$ remain, with the even terms in $B_{x}$ canceling each other out. Finally, to lowest order in $B_{x}$, we get

$$
\delta E=2 S \frac{B_{x}}{\Omega_{0}} \sum_{i \neq j} V_{i j}^{x z}\left\langle\Phi_{s}\left|S_{i}^{z}\right| \Phi_{s}\right\rangle .
$$

Taking the largest $V_{i j}^{x z}$ with a typical value $V_{\perp}$, the typical energy gained by flipping a droplet of $N \sim L^{d}$ spins is, to leading order in $B_{x}$,

$$
\langle|\delta E|\rangle \propto \frac{S^{2} B_{x} V_{\perp} \sqrt{N}}{\Omega_{0}},
$$

indicating that the total energy gain increases with $B_{x}$ linearly to leading order, as first found in Refs. 25 and 27.

This decrease in energy is to be compared with the energy cost due to the formation of a spin-glass droplet. ${ }^{29}$ This energy cost scales with the linear size $L$ of the droplet, $L$ $=N^{1 / 3}$, as $\approx S^{2} V_{\|} L^{\theta_{d}}$, where $V_{\|}$is the typical value of the largest $V_{i j}^{z z}$, which one typically expects to be of the same order as $V_{\perp}$. Comparing the energy gain $\langle|\delta E|\rangle$ of Eq. (5) with the energy cost for droplet formation, Refs. 25 and 27 find a finite correlation length $\xi$, identified with $L$, which, for small $B_{x}$, scales as

$$
\xi \approx\left(\frac{\Omega_{0} V_{\|}}{B_{x} V_{\perp}}\right)^{1 /\left(3 / 2-\theta_{d}\right)} .
$$

Based on an argument by Fisher and Huse, ${ }^{29} \theta_{d} \leqslant(d-1) / 2$ or $\theta_{d}<3 / 2$ here. Hence, turning on $B_{x}$ leads to a reduction of the correlation length $\xi\left(B_{x}\right)$, inhibiting its divergence, which occurs when $B_{x}=0$. In other words, the presence of the applied transverse $B_{x}$ leads, via the presence of the off-diagonal $V_{i j}^{x z}$ spin-spin interactions, to a destruction of the spin-glass phase with a typical spin-glass correlation length $\xi$ decreasing as $B_{x}$ increases. References 25 and 27 argue that this is the mechanism via which the nonlinear magnetic susceptibility $\chi_{3}$ no longer diverges in $\mathrm{LiHo}_{x} \mathrm{Y}_{1-x} \mathrm{~F}_{4}$ as $B_{x}$ is increased from zero. ${ }^{5,38}$ 


\section{EFFECTIVE SPIN- $\frac{1}{2}$ DESCRIPTION}

In the previous section we reviewed the arguments of Refs. 25 and 27 which lead to the key result of Eq. (4). We now proceed to show that a reformulation of the microscopic spin Hamiltonian, Eq. (1), in terms of an effective $S_{\text {eff }}=\frac{1}{2}$ model, leads identically to Eq. (4) in the limit of small $B_{x} / D$.

First, we focus on a situation where the temperature considered is low compared to $\Omega_{0}$, and project the spin $S$ operators onto the two-dimensional subspace formed by the two lowest-energy eigenstates $\left|\alpha\left(B_{x}\right)\right\rangle$ and $\left|\beta\left(B_{x}\right)\right\rangle$. Following Refs. 13 and 26, we define an Ising subspace $|\uparrow\rangle$ and $|\downarrow\rangle$ by performing a rotation

$$
\begin{aligned}
& |\uparrow\rangle=\frac{1}{\sqrt{2}}[|\alpha\rangle+\exp (i \theta)|\beta\rangle], \\
& |\downarrow\rangle=\frac{1}{\sqrt{2}}[|\alpha\rangle-\exp (i \theta)|\beta\rangle] .
\end{aligned}
$$

The phase $\theta$ is chosen such that the matrix elements of the operator $S^{z}$ within the new (Ising) subspace are real and diagonal. In this case, we can define $S_{i}^{z}=C_{z z} \sigma_{i}^{z}$. This allows us to recast $\mathcal{H}$ in Eq. (1) in terms of an effective spin- $\frac{1}{2}$ Hamiltonian $\mathcal{H}_{\text {eff }}$ that involves the $\sigma^{\mu}$ Pauli matrices. ${ }^{13}$ In this projected subspace, a transverse field $\Gamma=\frac{1}{2} \Delta\left(B_{x}\right)$ acts on the effective $\sigma_{i}^{x}$ spin. The projected $S_{i}^{\mu}(\mu=x, y, z)$ operator may be written as

$$
S_{i}^{\mu}=\sum_{\nu=1}^{\nu=3} C_{\mu \nu}\left(B_{x}\right) \sigma_{i}^{\nu}+C_{\mu 0}\left(B_{x}\right) 1 .
$$

The $C_{\mu \nu}$ and $\Delta$ dependence on $B_{x}$ can be obtained by exact diagonalization $^{13,26}$ of the noninteracting part of $\mathcal{H}$ (i.e., $\left.V_{i j}^{\mu \nu}=0\right)$ in Eq. (1).

For zero transverse field, $B_{x}=0$, the only nonzero $C_{\mu \nu}$ coefficient is $C_{z z}(0)=S$, giving a classical (effective) lowenergy dipolar Ising model

$$
\mathcal{H}_{\text {Ising }}=-\frac{1}{2} S^{2} \sum_{i \neq j} V_{i j}^{z z} \sigma_{i}^{z} \sigma_{j}^{z}
$$

Turning on $B_{x}$, the coefficients $C_{x 0}$ and $C_{x x}$ increase with $B_{x}$, while $C_{z z}$ shows a slight decrease with increasing $B_{x}$, as shown in Fig. 1. Thus, by substituting $S_{i}^{z}$ with $C_{z z}\left(B_{x}\right) \sigma_{i}^{z}$ and $S_{i}^{x}$ with $C_{x x}\left(B_{x}\right) \sigma_{i}^{x}+C_{x 0}\left(B_{x}\right) \rrbracket$ in Eq. (1), the effective spin- $\frac{1}{2}$ Hamiltonian is

$$
\begin{aligned}
\mathcal{H}_{\text {eff }}= & -\frac{1}{2} C_{z z}^{2}\left(B_{x}\right) \sum_{i \neq j} V_{i j}^{z z} \sigma_{i}^{z} \sigma_{j}^{z}-C_{z z}\left(B_{x}\right)\left(C_{x x}\left(B_{x}\right) \sum_{i \neq j} V_{i j}^{z x} \sigma_{i}^{z} \sigma_{j}^{x}\right. \\
& \left.-C_{x 0}\left(B_{x}\right) \sum_{i \neq j} V_{i j}^{z x} \sigma_{i}^{z}\right)-\frac{1}{2} \Delta\left(B_{x}\right) \sum_{i} \sigma_{i}^{x} .
\end{aligned}
$$

As can be seen, the projection of the $V_{i j}^{z x} S_{i}^{z} S_{j}^{x}$ term in Eq. (1) results in an induced random bilinear coupling, proportional to $\sigma_{i}^{z} \sigma_{j}^{x}$, and a longitudinal random field interaction, proportional to $\sigma_{i}^{z}$, for $B_{x} \neq 0$. For low enough transverse field $B_{x}$, the Ising dipolar interaction (proportional to $V_{i j}^{z z}$ ) is the dominant term.

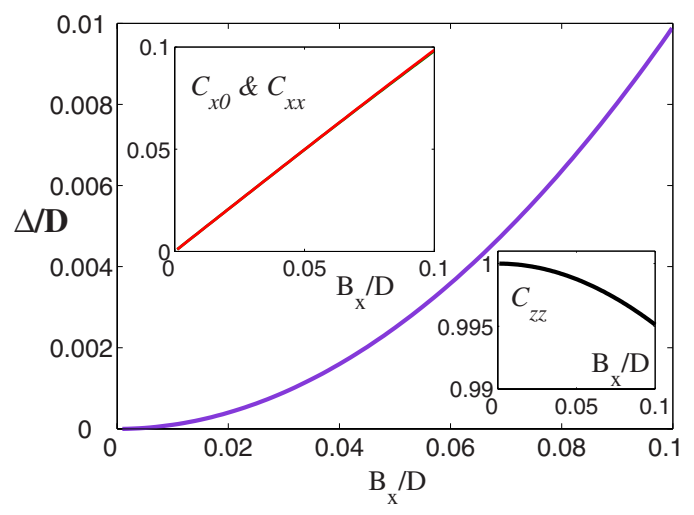

FIG. 1. (Color online) Evolution of $\Delta, C_{z z}, C_{x 0}$, and $C_{x x}$ as functions of the external transverse field $B_{x}$ for $S=1 . \Delta$ is the energy gap between the two lowest states $\langle\alpha\rangle$ and $\langle\beta\rangle$ in absence of interactions $\left(V_{i j}^{\mu \nu}=0\right) . C_{z z}, C_{x 0}$, and $C_{x x}$ are the nonzero coefficients used to map the matrix elements of the $\mathbf{S}=1$ spin operator within the low-energy manifold spanned by $|\alpha\rangle$ and $|\beta\rangle$ to that of an effective $S_{\text {eff }}=\frac{1}{2}$ operator.

Having derived the effective Hamiltonian, we now repeat the calculation of $\delta E$ within this effective $S_{\text {eff }}=\frac{1}{2}$ framework by again bringing in the spin-glass droplet picture. ${ }^{29}$ For $B_{x}$ $=0$, we denote by $|\psi\rangle$ the ground state of the $S_{\text {eff }}=\frac{1}{2}$ system where $|\psi\rangle$ is a specific realization of the $\uparrow$ and $\downarrow$ (effective) Ising spins configuration. ${ }^{39}$ For $B_{x}=0$, because of timereversal symmetry, the time-reversed state $|\tilde{\psi}\rangle$, which is obtained by flipping all the spins of $|\psi\rangle$, is a ground state of the system as well. We thus have a ground-state doublet in the droplet picture for the effective spin model. Carrying on a similar discussion as in the previous section and as in Refs. 25 and 27, at low enough $B_{x}$ within a droplet picture, the symmetry is broken due the presence of the induced random fields in Eq. (10). The energy cost to flip the spins over a droplet is

$$
\delta E \equiv\left\langle\widetilde{\psi}\left|H_{\mathrm{eff}}\right| \widetilde{\psi}\right\rangle-\left\langle\psi\left|H_{\mathrm{eff}}\right| \psi\right\rangle,
$$

which, to lowest order in $B_{x}$, gives

$$
\delta E \approx 2 C_{z z} C_{x 0} \sum_{i \neq j} V_{i j}^{x z}\left\langle\psi\left|\sigma_{i}^{z}\right| \psi\right\rangle
$$

Although we have an exact analytical expression for the $C_{\mu \nu}$ coefficients as a function of $B_{x}$ (which is available for $S$ $\leqslant 3 / 2$ ), in order to compare with Eq. (4) above and with Refs. 25 and 27, we consider the $B_{x}$ dependence of $C_{\mu \nu}$ to leading order in $B_{x} / D$. Using standard degenerate perturbation theory, for $S>1$ and up to second order in $B_{x}{ }^{40}$ the $|\uparrow\rangle$ and $|\downarrow\rangle$ defined in Eq. (7) are given by

$$
|\uparrow\rangle=\left(1-\frac{B_{x}^{2}}{4 \Omega_{0}^{2}} S\right)|S\rangle+\frac{B_{x}}{\Omega_{0}} \sqrt{\frac{S}{2}}|S-1\rangle,
$$




$$
|\downarrow\rangle=\left(1-\frac{B_{x}^{2}}{4 \Omega_{0}^{2}} S\right)|-S\rangle+\frac{B_{x}}{\Omega_{0}} \sqrt{\frac{S}{2}}|-S+1\rangle,
$$

recalling that $\Omega_{0}=(2 S-1) D$. Returning to Eq. (8), from which the $C_{\mu \nu}$ are obtained $\left[C_{z z}=\frac{1}{2}\left(\left\langle\uparrow\left|S^{z}\right| \uparrow\right\rangle-\left\langle\downarrow\left|S^{z}\right| \downarrow\right\rangle\right)\right.$ and $\left.C_{x 0}=\frac{1}{2}\left(\left\langle\uparrow\left|S^{x}\right| \uparrow\right\rangle+\left\langle\downarrow\left|S^{x}\right| \downarrow\right\rangle\right)\right]$, we use Eq. (12) to find $C_{z z}$ $\approx S\left(1-B_{x}^{2} / 2 \Omega_{0}^{2}\right), C_{x 0} \approx S B_{x} / \Omega_{0}, C_{x x} \propto\left(B_{x}\right)^{2 S-1}\left(C_{x x} \approx B_{x} / \Omega_{0}\right.$ for $S=1$ ), while $\Delta \propto\left(B_{x}\right)^{2 S}{ }^{36}$ Substituting those $B_{x}$ dependencies back in Eq. (11) and keeping terms to lowest order in $B_{x}$, the dependence of the energy cost $\delta E$ is

$$
\delta E \approx 2 S^{2} \frac{B_{x}}{\Omega_{0}} \sum_{i \neq j} V_{i j}^{x z}\left\langle\psi\left|\sigma_{i}^{z}\right| \psi\right\rangle .
$$

Referring to Eq. (12), we have $\left\langle\Phi\left|S_{i}^{z}\right| \Phi\right\rangle \approx S\left\langle\psi\left|\sigma_{i}^{z}\right| \psi\right\rangle$ to zeroth order in $B_{x}$. Hence, we find that the energy cost obtained in the $S_{\text {eff }}=\frac{1}{2}$ picture is identical to the energy cost given by Eq. (4) obtained via second-order perturbation theory and previously reported in Refs. 25 and 27. Thus, Eq. (13) leads to the same rms energy cost for flipping a droplet, given by Eq. (5), and the same $B_{x}$ dependence of the spin-glass correlation length $\xi$ in Eq. (6). Hence, we have shown that a formally derived effective $S_{\text {eff }}=1 / 2$ Hamiltonian does capture quantitatively the low-energy physics of the full $S$ Hamiltonian at low transverse fields. While the argument above was constructed for the toy model of Eq. (1), one could proceed identically for the full-blown microscopic Hamiltonian of $\mathrm{LiHo}_{x} \mathrm{Y}_{1-x} \mathrm{~F}_{4}$. Indeed, this is the underlying program carried in Ref. 26.

\section{NUMERICAL RESULTS}

In the same spirit as Refs. 25 and 27, we have performed numerical calculations to back up our perturbative approach. This allows us to investigate to what extent our proposed low-energy effective spin- $\frac{1}{2}$ model is a good description of the full anisotropic Hamiltonian (1) as well as determine the range of transverse field over which the above analytical small- $B_{x}$ results are valid. In this section we present results from exact diagonalizations on finite-size clusters with open boundary conditions. ${ }^{41}$ In order to compare the present approach with the previous investigations done by Schechter $e t$ $a l .{ }^{25,27}$ we work at the same constant dipole concentration $x=18.75 \%$.

$\mathrm{LiHoF}_{4}$ is a compound with space group $C_{4 h}^{6}\left(I 4_{1} / a\right)$ with lattice parameters $a=b=5.175 \AA, c=10.75 \AA$, and with four $\mathrm{Ho}^{3+}$ ions per unit cell positioned at $(0,0,1 / 2)$, $(0,1 / 2,3 / 4), \quad(1 / 2,1 / 2,0), \quad$ and $(1 / 2,0,1 / 4) .{ }^{43}$ For $\mathrm{LiHo}_{x} \mathrm{Y}_{1-x} \mathrm{~F}_{4}$, a dilution of $x=18.75 \%$ is realized by distributing randomly $N$ magnetic moments (holmium $\mathrm{Ho}^{3+}$ ions) in a sample of $16 N / 3$ possible sites. We have chosen samples of size $(2 a, 2 b, c N / 3)$, where $N$ is a multiple of 3 . Thus, changing the number $N$ of magnetic ions means changing the size of the sample in the $z$ direction in order to keep a constant dilution.

In Eq. (1), the dipolar interaction is written as $V_{i j}^{\mu \nu}$, which takes, with the negative coefficient convention used in Eq. (1), the explicit form

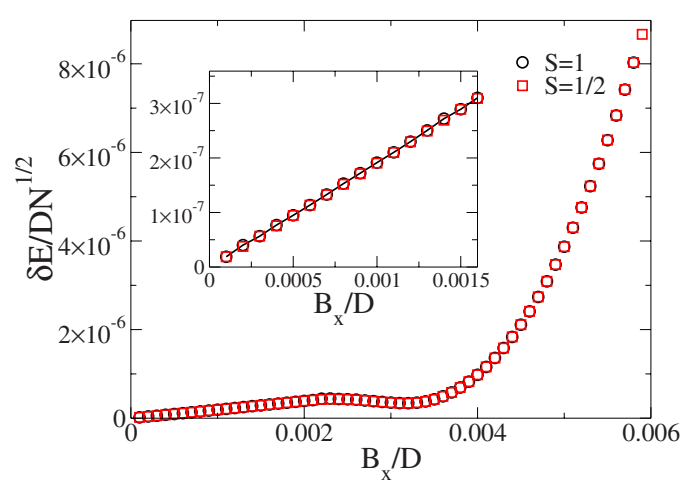

FIG. 2. (Color online) Comparison between the $S=1$ and $S_{\text {eff }}$ $=1 / 2$ models for a given sample (e.g., realization of disorder) of $N=9$ spins: gap $\delta E /(D \sqrt{N})$ as a function of the transverse field $B_{x} / D . \delta E$ is the energy gap between the two lowest-energy states of the system in presence of both dipolar interactions $V_{i j}^{\mu \nu}$ and the transverse magnetic field $B_{x}$. The inset shows the small- $B_{x} / D$ regime for the same sample.

$$
V_{i j}^{\mu \nu}=\frac{\mu_{B}^{2}}{r_{i j}^{3}}\left(\frac{3 r_{i j}^{\mu} r_{i j}^{\nu}}{r_{i j}^{2}}-\delta_{\mu \nu}\right),
$$

where $r_{i j}$ is the distance between the ions at positions $i$ and $j$, and $\mu, \nu=x, y, z$. The dipolar interaction $V^{z z}$ is of the order $\mu_{B}^{2} / a^{3} \approx 4.49 \times 10^{-3} \mathrm{~K}$, whereas the on-site anisotropy is taken as $D=10 \mathrm{~K}$. In the following, we investigate the behavior of the gap $\delta E$ between the ground state and first excited state as a function of the applied transverse field $B_{x}$. Since we are mainly interested in checking the relations (4), (5), and (13), we present our results in terms of renormalized parameters $\left(\delta E / D \sqrt{N}, B_{x} / D\right)$.

To perform a first check of the validity of our approach, we choose a small cluster with a fixed random distribution of $N=9$ spins and compute the renormalized gap $\delta E /(D \sqrt{N})$ for both models [i.e., $S=1$, Eq. (1), and $S_{\text {eff }}=\frac{1}{2}$, Eq. (10)] as a function of the reduced transverse magnetic field $B_{x} / D$. The results are shown in Fig. 2. In zero transverse field the ground state is degenerate and its energetics are governed by the Ising interaction $V^{z z}$. The application of a transverse field $B_{x}$ lifts the degeneracy. The splitting energy between the ground state and the first excited state corresponds to excitation energy $\delta E$ between the ground state and the excited state with spins flipped. For sufficiently small $B_{x}$, the most important interaction remains $V^{z z}$, and the gap $\delta E$ is found to be proportional to $B_{x} / D$ (inset of Fig. 2), as suggested by the arguments leading to Eqs. (4) and (13). Upon increasing $B_{x} / D$ to a sufficiently large value, the transverse field eventually becomes stronger than the dipolar interactions. At that point, the perturbative low- $B_{x}$ regime ${ }^{44}$ is no longer valid and the gap $\delta E$ is no longer proportional to $B_{x}$. However, Fig. 2 shows that, even for high transverse fields, we observe an almost exact agreement between the numerical results for the $S=1$ model and the effective $S_{\text {eff }}=\frac{1}{2}$ description.

Interestingly, for a specific realization of disorder, in Fig. 2 , we note a local maximum in $\delta E$ around $B_{x} / D \approx 0.0024$, followed by a local minimum, before $\delta E$ starts diverging 


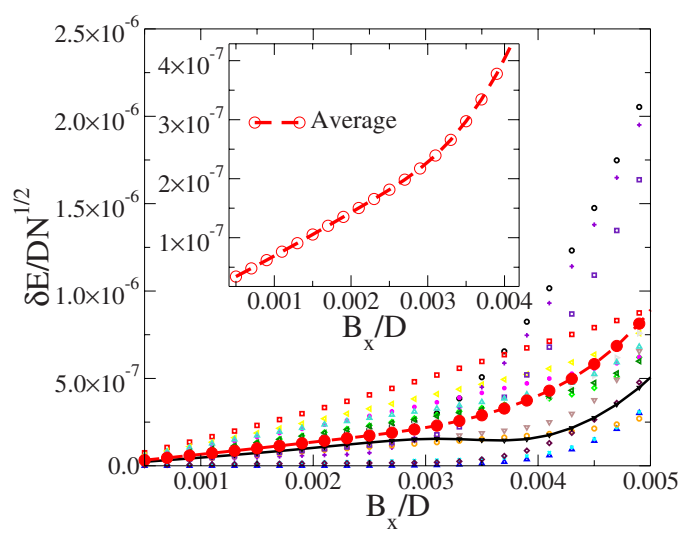

FIG. 3. (Color online) Random variations of the disorder configurations for an $N=6$ spin system for 20 realizations of disorder. Gap $\delta E /(D \sqrt{N})$ as a function of the transverse field $B_{x} / D$. Depending on the disorder configuration, the curves exhibit a local maximum and a local minimum. The thin (black) curve in the main panel shows the minimum-maximum structure of $\delta E$ vs $B_{x}$ for a specific realization of disorder. This structure disappears after taking the average, as shown by the thick (red) curve joining the filled (red) circles. The monotonic behavior for the average of $\delta E$, already for 20 samples, is emphasized in the inset.

with increasing $B_{x}$. We investigated the origin of this behavior and found that it can be understood as arising from the $B_{x}$ dependence of $C_{z z} \propto\left[1-\frac{1}{2}\left(B_{x} / \Omega_{0}\right)^{2}\right]$ vs $C_{x 0} \propto B_{x} / \Omega_{0}$, both for small $B_{x} / D$. The dependence of $\delta E$ on $B_{x}$ is controlled not only by the $C_{\mu \nu}$ parameters, but also by a prefactor coming from the dipolar interactions, which is proportional to $r_{i j}^{z} r_{i j}^{x}$. Obviously, if this prefactor is of relevance to the existence of the above maximum/minimum features, the random distribution of the magnetic ions in the sample must play a crucial role in the position (and even the existence) of this local maximum or minimum feature. If one takes an extreme case in which all the magnetic ions are aligned on a line along the $\hat{z}$ direction, the resultant interaction is 0 , and there is no dip in the curve. To confirm this scenario we show in Fig. 3 $\delta E /(D \sqrt{N})$ as a function of the transverse field $B_{x}$ for 20 different disorder configurations for $N=6$. One sees that the majority of curves do not show these local maximum and minimum features and, as shown by the inset of Fig. 3, the average of $\delta E$ over those 20 realizations of disorder reveals no such maximum-minimum structure.

Having demonstrated the one-to-one correspondence between the $S=1$ and the effective $S_{\text {eff }}=\frac{1}{2}$ model for various (specific) realizations of disorder, we now proceed to check the scaling with system size for $\langle|\delta E|\rangle$ predicted by Eq. (5) for the $S=1$ model, and also check that it agrees with that for the effective $S_{\text {eff }}=\frac{1}{2}$ model. The results for both models are shown in Fig. 4. The average gap $\langle|\delta E|\rangle$ was computed over 1000 samples which, for each system size of $N$ spins, we renormalize as $\langle|\delta E|\rangle /(D \sqrt{N})$, and plot for both models $(S$ $=1$ and $S_{\text {eff }}=\frac{1}{2}$ ) as a function of the transverse field $B_{x}$. As predicted by Eq. (5) and previously found in Ref. 25, our numerical results displayed in Fig. 4 reveal a regime for which the spin $S=1$ model (closed symbols) obeys $\langle|\delta E|\rangle / D \sqrt{N} \propto B_{x} / D$ scaling.

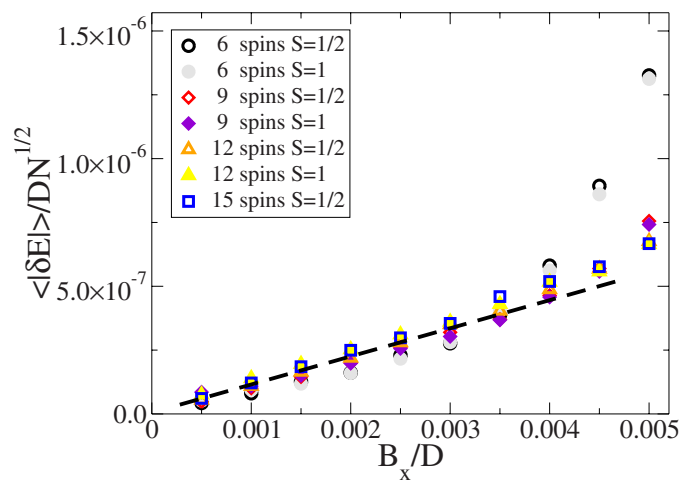

FIG. 4. (Color online) Scaling of the renormalized gap $\langle\delta E\rangle / \sqrt{N}$ (average taken over 1000 samples) for various system sizes as a function of the transverse field $B_{x}$. The closed symbols are for the $S=1$ model and the open symbols are for the effective $S_{\text {eff }}=\frac{1}{2}$ Hamiltonian.

One can see that at higher $B_{x}$ the scaling relation for different system size $N$, as well as the proportionality of the gap $\langle|\delta E|\rangle$ with $B_{x}$, starts to break down. As explained above in the context of Fig. 2, this comes from the fact that the transverse field term in the Hamiltonian is larger than the dipolar interaction $V_{i j}^{z z}$. Thus the droplet picture is no longer valid, and the scaling and proportionality relations in Eq. (5) are no longer satisfied. We also show in Fig. 4 the results for the effective $S_{\text {eff }}=\frac{1}{2}$ model (open symbols), demonstrating the agreement with the results for the $S=1$ model, even when the $\delta E / \sqrt{N} \propto B_{x} / D$ regime breaks down. This confirms the correctness of the conclusion based on Eq. (13) when compared with Eq. (4), and that $\delta E$ is the same for both the $S=1$ and the $S_{\text {eff }}=1 / 2$ models.

\section{CONCLUSION}

We have shown how to rigorously derive an effective spin- $\frac{1}{2}$ Hamiltonian to describe the problem of induced random fields in a spin-glass model with strong single-ion Ising anisotropy and subject to a transverse magnetic field. We discussed the relation of this model with the physics of the $\mathrm{LiHo}_{x} \mathrm{Y}_{1-x} \mathrm{~F}_{4}$ material in a magnetic field transverse to the $\mathrm{Ho}^{3+}$ Ising spins. ${ }^{5}$ We have shown, both analytically and numerically, that the use of such a model gives results in full quantitative agreement with previously reported perturbation theory calculations on a "large" spin- $S$ toy model with strong anisotropy. ${ }^{25,27,28}$ However, the large hyperfine interactions present in the real $\mathrm{LiHo}_{x} \mathrm{Y}_{1-x} \mathrm{~F}_{4}$, and which have been ignored here, must ultimately be considered in order to obtain a good quantitative understanding of the low-temperature regime. $^{35}$

The approach of Refs. 25 and 27 proceeds via RayleighSchrödinger perturbation theory, while the approach in Ref. 26 and presented in Sec. III above relies on the effective Hamiltonian approach. To low order in the quantum $\mathcal{H}_{\perp}$ term, the two approaches have been shown to give identical results. However, the emergence of induced random fields is perhaps more apparent in the spin- $\frac{1}{2}$ effective model ap- 
proach. The $C_{\mu \nu}$ coefficients needed to construct the effective Hamiltonian are easily calculated, providing the ability to investigate the evolution of $\delta E$ and $\xi$ with $B_{x}$ beyond the linear term and to arbitrarily high order in $B_{x}$. Such highorder perturbation theory would be more cumbersome to construct when proceeding via a direct Rayleigh-Schrödinger perturbation scheme. In this context, the crucial step connecting the perturbation theory method and the effective $S_{\text {eff }}=\frac{1}{2}$ approach is in the determination of the $B_{x}$ dependence of the $C_{\mu \nu}$ transformation parameters in Eq. (8). It is the neglect of this $B_{x}$ dependence of the spin interactions in the $S_{\text {eff }}=\frac{1}{2}$ model that leads to the different results for the $S_{\text {eff }}$ $=\frac{1}{2}$ and $S=1$ models investigated in Ref. 27, and which led their authors to argue for the quantitative inadequacies of the $S_{\text {eff }}=\frac{1}{2}$ approach. Per se, the numerical results for the $S_{\text {eff }}$ $=\frac{1}{2}$ and $S=1$ models reported in Ref. 27 are correct. Indeed, there is no problem in finding a different $B_{x}$ dependence of the disorder average $|\delta E|$ if one is not interested in making a formal connection between the $S=1$ and the $S_{\text {eff }}=\frac{1}{2}$ model. However, in a study aiming at calculating physical quantities defined in the high-energy sector, one may want, as we do in Ref. 26 and in the present paper, to formally relate the "microscopic" (bare) high-energy model to the effective lowenergy description.

We note that, in a general case where the $V_{i j}^{z x}$ spin-spin interactions are not much smaller than $D$, higher-order perturbation theory calculations must be carried out to derive an effective Hamiltonian. The physical result would be that virtual transitions to the excited states lead to an admixing of those states with the low-energy sector. This effect was recently discussed in Ref. 45, where it was shown that such effective interaction-induced quantum mechanical effects are seemingly negligible for $\mathrm{LiHo}_{x} \mathrm{Y}_{1-x} \mathrm{~F}_{4}$. This makes it difficult to justify the idea of quantum mechanical entanglement to explain the peculiar behavior of the very dilute $\mathrm{LiHo}_{x} \mathrm{Y}_{1-x} \mathrm{~F}_{4}$ $(x=0.045)$ as advocated in Ref. 7. However, as a counterexample and for a different magnetic rare-earth system, we note that it was recently found that such interaction-induced admixing can dramatically change the low-energy physics. ${ }^{46}$

With the contributions of Refs. 25-28 and 35 and the clarification presented herein, it may be that the behavior of dilute $\mathrm{LiHo}_{x} \mathrm{Y}_{1-x} \mathrm{~F}_{4}$ in a transverse field, in both the random ferromagnetic and spin-glass regimes, is now somewhat un- derstood. This impression would seem to be further corroborated by recent experimental studies, which provide evidence for the manifestation of induced random fields for $\mathrm{LiHo}_{x} \mathrm{Y}_{1-x} \mathrm{~F}_{4}$ with $x=0.44$ and $B_{x}>0 .{ }^{9,47}$ Yet there are many questions still open regarding the physics of this material for $x \leqslant 20 \%$ : Is there a dipolar spin glass phase over a reasonably wide range of dipole moment concentration, either theoretically, 78,49 or experimentally? $?^{4,7,17,50}$ What are the physical objects giving rise to the peculiar coherent dynamics at low temperature for samples with low $\mathrm{Ho}^{3+}$ concentration (see Refs. 6 and 10)? Even for pure $\mathrm{LiHoF}_{4}$, what is the microscopic explanation for the discrepancy between experimental results and Monte Carlo simulations for the temperature vs transverse-field phase diagram for small $B_{x}$ near the classical paramagnetic phase boundary? ${ }^{13,42}$ Are the phenomena found in zero and nonzero $B_{x}$ for $\mathrm{LiHo}_{x} \mathrm{Y}_{1-x} \mathrm{~F}_{4}$ also observable in other Ising systems which possess either Kramers or non-Kramers rare-earth magnetic ions? ${ }^{22}$ How do similar diluted dipolar Ising systems, with hyperfine interactions of much lesser importance than for $\mathrm{Ho}^{3+}$ ions, ${ }^{33-35}$ behave at low temperatures? While it is interesting that $\mathrm{LiHo}_{x} \mathrm{Y}_{1-x} \mathrm{~F}_{4}$ in a transverse field becomes a rare, if not the first physical realization of a random-field Ising model in a ferromagnetic setting, $, 26,28,32,47$ it would seem that this is a small part of the challenges offered by this material, with apparently more left to understand than has so far been understood.

Note added in proof. Recently, experimental results on the putative spin glass transition in $\mathrm{LiHo}_{x} \mathrm{Y}_{1-x} \mathrm{~F}_{4}$ and exploring the competing effects of quantum entanglement and random fields in this material, both induced by a transverse field, were reported by C. Ancona-Torres et al. ${ }^{51}$

\section{ACKNOWLEDGMENTS}

We thank Steve Girvin, Rob Hill, Helmut Katzgraber, and Pawel Stasiak for useful discussions, and Nicolas Laflorencie for comments on a previous version of this manuscript. Support for this work was provided by NSERC of Canada, the Canada Research Chair Program (Tier I, M.G), the Canada Foundation for Innovation, the Ontario Innovation Trust, and the Canadian Institute for Advanced Research. M.G. acknowledges the University of Canterbury (UC) for financial support and the hospitality of the Department of Physics and Astronomy at UC where part of this work was completed.
${ }^{1}$ A. H. MacDonald, S. M. Girvin, and D. Yoshioka, Phys. Rev. B 37, 9753 (1988); 41, 2565 (1990); 43, 6209 (1991).

${ }^{2}$ A. L. Chernyshev, D. Galanakis, P. Phillips, A. V. Rozhkov, and A.-M. S. Tremblay, Phys. Rev. B 70, 235111 (2004).

${ }^{3}$ J.-Y. P. Delannoy, M. J. P. Gingras, P. C. W. Holdsworth, and A.-M. S. Tremblay, Phys. Rev. B 72, 115114 (2005).

${ }^{4}$ D. H. Reich, B. Ellman, J. Yang, T. F. Rosenbaum, G. Aeppli, and D. P. Belanger, Phys. Rev. B 42, 4631 (1990).

${ }^{5}$ W. Wu, D. Bitko, T. F. Rosenbaum, and G. Aeppli, Phys. Rev. Lett. 71, 1919 (1993).

${ }^{6}$ S. Ghosh, R. Parthasarathy, T. F. Rosenbaum, and G. Aeppli,
Science 296, 2195 (2002).

${ }^{7}$ S. Ghosh, T. F. Rosenbaum, G. Aeppli, and S. N. Coppersmith, Nature (London) 425, 48 (2003).

${ }^{8}$ H. M. Rønnow, R. Parthasarathy, J. Jensen, G. Aeppli, T. F. Rosenbaum, and D. F. McMorrow, Science 308, 389 (2005).

${ }^{9}$ D. M. Silevitch, D. Bitko, J. Brooke, S. Ghosh, G. Aeppli, and T. F. Rosenbaum, Nature (London) 448, 567 (2007).

${ }^{10}$ D. M. Silevitch, C. M. S. Gannarelli, A. J. Fisher, G. Aeppli, and

T. F. Rosenbaum, Phys. Rev. Lett. 99, 057203 (2007).

${ }^{11}$ P. E. Hansen, T. Johansson, and R. Nevald, Phys. Rev. B 12, 5315 (1975). 
${ }^{12}$ H. M. Rønnow, J. Jensen, R. Parthasarathy, G. Aeppli, T. F. Rosenbaum, D. F. McMorrow, and C. Kraemer, Phys. Rev. B 75, 054426 (2007).

${ }^{13}$ P. B. Chakraborty, P. Henelius, H. Kjonsberg, A. W. Sandvik, and S. M. Girvin, Phys. Rev. B 70, 144411 (2004).

${ }^{14}$ D. Bitko, T. F. Rosenbaum, and G. Aeppli, Phys. Rev. Lett. 77, 940 (1996)

${ }^{15}$ An exotic "antiglass" state, whose nature is not yet well understood, occurs at low concentration $x$ of $\mathrm{Ho}^{3+}$. See Refs. 6, 7, and 10 .

${ }^{16} \mathrm{We}$ note that the existence of a spin-glass transition in $\mathrm{LiHo}_{x} \mathrm{Y}_{1-x} \mathrm{~F}_{4}(x=0.167)$ in zero transverse magnetic field has very recently been questioned. See Ref. 17.

${ }^{17}$ P. E. Jansson, R. Mathieu, W. Wernsdorfer, A. Tkachuk, and B. Barbara, Phys. Rev. Lett. 98, 256403 (2007).

${ }^{18}$ P. G. de Gennes, Solid State Commun. 1, 132 (1963).

${ }^{19}$ R. J. Elliott, P. Pfeuty, and C. Wood, Phys. Rev. Lett. 25, 443 (1970).

${ }^{20}$ S. Sachdev, Quantum Phase Transitions (Cambridge University Press, Cambridge, England, 1999).

${ }^{21}$ B. K. Chakrabarti, A. Dutta, and P. Sen, Quantum Ising Phases and Transitions in Transverse Ising Models (Springer-Verlag, Heidelberg, 1996).

${ }^{22} \mathrm{~A}$ recent theoretical study finds that the $\mathrm{Ho}(\mathrm{OH})_{3}$ and $\mathrm{Dy}(\mathrm{OH})_{3}$ uniaxial dipolar ferromagnets may constitute a new class of suitable materials to investigate the problem of transverse field physics in an Ising system. P. Stasiak and M. J. P. Gingras (unpublished).

${ }^{23}$ J. A. Mydosh, Spin Glasses: An Experimental Introduction (Taylor \& Francis, London, 1993).

${ }^{24}$ L. F. Cugliandolo, D. R. Grempel, and C. A. da Silva Santos, Phys. Rev. Lett. 85, 2589 (2000).

${ }^{25}$ M. Schechter and N. Laflorencie, Phys. Rev. Lett. 97, 137204 (2006).

${ }^{26}$ S. M. A. Tabei, M. J. P. Gingras, Y.-J. Kao, P. Stasiak, and J.-Y. Fortin, Phys. Rev. Lett. 97, 237203 (2006).

${ }^{27}$ M. Schechter, P. C. E. Stamp, and N. Laflorencie, J. Phys.: Condens. Matter 19, 145218 (2007).

${ }^{28}$ M. Schechter, Phys. Rev. B 77, 020401(R) (2008).

${ }^{29}$ D. S. Fisher and D. A. Huse, Phys. Rev. Lett. 56, 1601 (1986).

${ }^{30}$ Y. Imry and S. K. Ma, Phys. Rev. Lett. 35, 1399 (1975).

${ }^{31}$ The argument of Ref. 29 regarding the absence of a spin-glass transition in a magnetic field pertains to the case of a uniform magnetic field. However, the same conclusion applies to the case of a random field as long as the distribution of couplings is such that one is deep in the spin-glass regime and that the randomness in the sign of the random field can be "gauged away." In this context, a recent Monte Carlo study of the Ising spin glass specifically considered the case of random fields as opposed to a uniform field in order to check for the existence of a spin-glass transition in nonzero random field (and the presence of an Almeida-Thouless line) for a three-dimensional Ising spin glass model [see A. P. Young and H. G. Katzgraber, Phys. Rev. Lett. 93, 207203 (2004)].

${ }^{32}$ J. Brooke, Ph.D. thesis, University of Chicago, 2000.

${ }^{33}$ A. P. Ramirez and J. Jensen, J. Phys.: Condens. Matter 6, L215
(1994).

${ }^{34}$ S. T. Bramwell et al., Phys. Rev. Lett. 87, 047205 (2001).

${ }^{35}$ M. Schechter and P. C. E. Stamp, Phys. Rev. Lett. 95, 267208 (2005).

${ }^{36}$ For $S=1$, using second-order degenerate perturbation theory, one finds that $\Delta=B_{x}^{2}\left\langle 1\left|S^{x}\right| 0\right\rangle\left\langle 0\left|S^{x}\right| 1\right\rangle / \Omega_{0}$. For $S>1$, one should go to higher orders of perturbation theory, obtaining the leading term proportional to $\left(B_{x}\right)^{2 S}$

${ }^{37}$ K. T. Hecht, Quantum Mechanics (Springer-Verlag, New York, 2000).

${ }^{38}$ Reference 26 also found, using a replica-symmetric mean-field theory calculation (in the static limit), that $\chi_{3}$ becomes immediately nonsingular as $B_{x}$ is turned on. However, as that calculation proceeds within a mean-field framework, it makes no comment as to the existence or not of a thermodynamic spin-glass transition in the presence of nonzero $B_{x}$ in a real threedimensional system.

${ }^{39}$ For $B_{x}=0,|\uparrow\rangle=|S\rangle$ and $|\downarrow\rangle=|-S\rangle$, which indicates that, at $B_{x}=0$, $|\psi\rangle=\left|\Phi_{S}\right\rangle$.

${ }^{40}$ For $\quad S=1, \quad|\uparrow\rangle=\left(1-B_{x}^{2} / 4 \Omega_{0}^{2}\right)|1\rangle+\left(B_{x} / \Omega_{0} \sqrt{2}\right)|0\rangle-\left(B_{x}^{2} / 4 \Omega_{0}^{2}\right)|-1\rangle$ and $|\downarrow\rangle=\left(1-B_{x}^{2} / 4 \Omega_{0}^{2}\right)|-1\rangle+\left(B_{x} / \Omega_{0} \sqrt{2}\right)|0\rangle-\left(B_{x}^{2} / 4 \Omega_{0}^{2}\right)|1\rangle$, but the final $B_{x}$ dependence of $C_{\mu \nu}$ 's is, to leading order, not changed, and is similar to that in the $S>1$ case.

${ }^{41}$ Since our goal is merely to compare numerically the energy eigenvalues of two quantum spin models, as opposed to describing a system approaching the thermodynamic limit, we consider here the simplest case of open boundary conditions as opposed to periodic boundary conditions, which could be implemented, for example, using Ewald summation techniques as done in Refs. 22, 26, and 42.

${ }^{42}$ S. M. A. Tabei, M. J. P. Gingras, Y.-J. Kao, and T. Yavors'kii, arXiv:0801.0443 (unpublished).

${ }^{43}$ G. Mennenga, L. J. de Jongh, and W. J. Huiskamp, J. Magn. Magn. Mater. 44, 59 (1984).

${ }^{44}$ This low- $B_{x}$ regime corresponds to a region where the transverse field $B_{x}$ is small compared to the longitudinal part of the dipolar interactions, $V^{z z}$.

${ }^{45}$ In a recent work, Chin and Eastham derived an effective two-state Hamiltonian for $\mathrm{LiHo}_{x} \mathrm{Y}_{1-x} \mathrm{~F}_{4}$, but for $B_{x}=0$. In that work, the effect of admixing of the crystal field levels via the spin interactions was investigated via a second order perturbation theory [A. Chin and P. R. Eastham, arXiv:cond-mat/0610544 (unpublished)].

${ }^{46}$ A similar approach to that employed in Ref. 45 was recently used to investigate the role of quantum fluctuations in the highly frustrated $\mathrm{Tb}_{2} \mathrm{Ti}_{2} \mathrm{O}_{7}$ pyrochlore antiferromagnet [H. R. Molavian, M. J. P. Gingras, and B. Canals, Phys. Rev. Lett. 98, 157204 (2007)].

${ }^{47}$ See also Z. Fisk, Nature (London) 48, 546 (2007).

${ }^{48}$ J. Snider and C. C. Yu, Phys. Rev. B 72, 214203 (2005).

${ }^{49}$ A. Biltmo and P. Henelius, Phys. Rev. B 76, 054423 (2007).

${ }^{50}$ J. A. Quilliam, C. G. A. Mugford, A. Gomez, S. W. Kycia, and J. B. Kycia, Phys. Rev. Lett. 98, 037203 (2007).

${ }^{51}$ C. Ancona-Torres, D. M. Silevitch, G. Aeppli, and T. F. Rosenbaum, arXiv:0801.2181 (unpublished). 